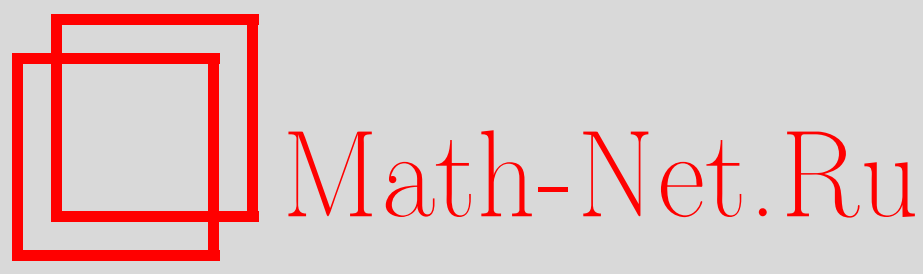

Г. А. Калагов, М. Ю. Налимов, М. В. Компаниец, Ренормгрупповое исследование сверхпроводящего фазового перехода: асимптотика высоких порядков разложений и результаты трехпетлевых расчетов, ТМФ, 2014, том 181, номер 2, 374-386

DOI: https://doi.org/10.4213/tmf8710

Использование Общероссийского математического портала Math-Net.Ru подразумевает, что вы прочитали и согласны с пользовательским соглашением http://www . mathnet.ru/rus/agreement

Параметры загрузки:

IP: 52.23 .180 .231

26 апреля 2023 г., 13:44:03

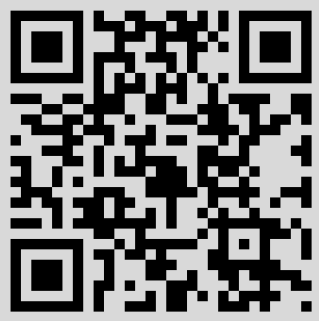




\title{
ФИЗИКА
}

Том 181, № 2

ноябрь, 2014

(C) 2014 г. . А. Калагов, М. В. Компаниец, М. Ю. Налимов

\section{РЕНОРМГРУППОВОЕ ИССЛЕДОВАНИЕ СВЕРХПРОВОДЯЩЕГО ФАЗОВОГО ПЕРЕХОДА: АСИМПТОТИКА ВЫСОКИХ ПОРЯДКОВ РАЗЛОЖЕНИЙ И РЕЗУЛЬТАТЫ ТРЕХПЕТЛЕВЫХ РАСЧЕТОВ}

\begin{abstract}
Методами квантово-полевой ренормализационной группы исследован фазовый переход в равновесной системе нерелятивистских ферми-частиц с взаимодействием типа "плотность-плотность" в формализме температурных функций Грина. Основное внимание уделено случаю частиц со спином больше $1 / 2$ или фермионных полей, имеющих по тем или иным причинам дополнительные индексы. В окрестности точки фазового перехода данная модель сведена к теории типа $\phi^{4}$ с матричным комплексным антисимметричным полем. Определено семейство инстантонов данной модели, исследовано асимптотическое поведение квантово-полевых разложений в ней. Выполнены трехпетлевые расчеты $\beta$-функций уравнения ренормализационной группы в $(4-\epsilon)$-схеме. В физических размерностях пространства $D=2,3$ произведено пересуммирование решений уравнений ренормализационной группы на траектории инвариантных зарядов. Результаты подтверждают сделанное ранее предположение о том, что в рассматриваемой системе имеет место переход в сверхпроводящее состояние первого рода, причем при более высокой температуре, чем это предсказывается классической теорией.
\end{abstract}

Ключевые слова: температурные функции Грина, сверхпроводимость, ренормализационная группа, инстантонный анализ, борелевское суммирование.

DOI: $10.4213 / \operatorname{tmf} 8710$

\section{1. ВВЕДЕНИЕ}

В работе [1] в формализме температурных функций Грина исследовалась квантовая равновесная система нерелятивистских фермионов. Анализ основывался на микроскопической модели с бесструктурным локальным взаимодействием типа 
“плотность-плотность" [2], [3]. Действие модели имеет вид

$$
S=\psi^{+}\left(\partial_{t}-\frac{\Delta}{2 m}-\mu\right) \psi-\frac{\lambda}{2}\left(\psi^{+} \psi\right)^{2},
$$

где $\psi=\left(\psi_{1}, \psi_{2}, \ldots, \psi_{r}\right)^{\mathrm{T}}, \psi^{+}=\left(\psi_{1}^{*}, \psi_{2}^{*}, \ldots, \psi_{r}^{*}\right)$ описывают фермионное поле при температуре $T$, компоненты $\psi_{i}, \psi_{i}^{*}$ - комплексно-сопряженные элементы грассмановой алгебры, зависимость полей от $D$-мерных координат и "мнимого времени" $t \in[0,1 / T]$, а также соответствующие интегрирования подразумеваются, $m$ - масса фермиона, $\Delta$ - оператор Лапласа, $\mu$ - химический потенциал системы, $\lambda$ - положительная константа связи. На поля $\psi, \psi^{+}$налагаются антипериодические граничные условия по $t$. Обычно носителями заряда в системах, демонстрирующих переход в сверхпроводящее состояние, являются электроны проводимости, им соответствует значение $r=2$. Однако в работе [1] рассмотрен случай произвольного четного $r$. При этом речь может идти как о носителях заряда с высшими спинами, так и о появлении у обычных электронных полей дополнительных индексов, например индекса подрешетки и (или) индекса, соответствующего вырождению зонной структуры, как это имеет место при рассмотрении электропроводности в графенах [4].

С помощью преобразования типа Хаббарда-Стратоновича в работе [1] были введены бозонные поля $\chi, \chi^{+}$, в новых переменных действие модели принимает вид

$$
S=\psi^{+}\left(\partial_{t}-\frac{\Delta}{2 m}-\mu\right) \psi+\operatorname{Tr} \chi \chi^{+}+\sqrt{\frac{\lambda}{2}} \psi_{i}^{*} \chi_{i j} \psi_{j}^{*}+\sqrt{\frac{\lambda}{2}} \psi_{i} \chi_{j i}^{*} \psi_{j} .
$$

Действительно, гауссово интегрирование функции $e^{-S}$ с действием $(2)$ по полям $\chi, \chi^{+}$ возвращает нас к действию (1). Поля $\psi, \psi^{+}$грассмановы, поэтому поля $\chi, \chi^{+}$можно рассматривать как комплексные антисимметричные матрицы ранга $r$. Эти поля, как показано в работе [1] с помощью уравнений Швингера

$$
\begin{aligned}
& \left\langle\chi_{i j}^{*}-\sqrt{\frac{\lambda}{2}} \psi_{i}^{*} \psi_{j}^{*}\right\rangle=\left\{A_{\chi^{+}}\right\}_{i j}, \\
& \left\langle\chi_{j i}+\sqrt{\frac{\lambda}{2}} \psi_{i} \psi_{j}\right\rangle=\left\{A_{\chi}\right\}_{i j},
\end{aligned}
$$

где $A$ обозначает соответствующий источник, связаны с параметром порядка, а в точке перехода являются безмассовыми критическими модами. Интегрирование по полям $\psi, \psi^{+}$в модели (2) позволяет построить эффективное действие $S_{\mathrm{e}}$ в терминах бозонных полей $\chi, \chi^{+}$:

$$
S_{\mathrm{e}}=\operatorname{Tr} \chi \chi^{+}+\frac{1}{2} \frown *\left(\frac{1}{4} \sim+\cdots+A_{\psi} G A_{\psi^{+}}\right. \text {, }
$$

где волнистыми хвостиками обозначены поля $\chi, \chi^{+}$, линии обозначают пропагаторы $\left\langle\psi \psi^{+}\right\rangle$, крестик на линии соответствует полю с крестом, в петлевых вкладах в действие (3) подразумевается операция взятия следа по индексам. Существенную для дальнейшего анализа часть эффективного действия можно записать в виде гамильтониана Гинзбурга-Ландау [1]

$$
S_{\mathrm{e}}=\operatorname{Tr} \chi^{+}(-\Delta+\tau) \chi+\frac{g_{1}}{4}\left(\operatorname{Tr} \chi \chi^{+}\right)^{2}+\frac{g_{2}}{4} \operatorname{Tr} \chi \chi^{+} \chi \chi^{+} .
$$


Заметим, что взаимодействие с зарядом $g_{1}$ включено в действие (4) для мультипликативной ренормируемости теории. Несложно убедиться, что соответствующие контрчлены возникают в процессе ренормировки теории начиная с простейшей однопетлевой диаграммы. Интегрирование в этом действии осуществляется лишь по $D$-мерным координатам, поскольку в критической области поля $\chi, \chi^{+}$не зависят от $t$, а соответствующие интегралы порождают множитель $1 / T$.

Критерий устойчивости действия (4) (условие положительной определенности члена взаимодействия) можно сформулировать в виде неравенства

$$
g_{2}+r g_{1}>0
$$

при физических значениях $g_{2}>0$. Для фермионов с $r=4,6, \ldots$ члены действия (4) с коэффициентами $g_{1}, g_{2}$ линейно независимы, однако при $r=2$ между ними можно установить связь: при этом константы взаимодействия собираются в единый параметр $g=2 g_{1}+g_{2}$, а модель (4) сводится к обычной $O(2)$-симметричной теории $\phi^{4}$. Для проверки результатов наших вычислений мы рассматривали также случай $r=3$, при этом модель (4) эквивалентна $O(6)$ - $\phi^{4}$-модели.

Теория (4) ренормируется мультипликативно. В работе [1] инфракрасное (ИК) поведение данной теории было исследовано методом ренормализационной группы $(\mathrm{P})$. В однопетлевом приближении установлено, что модель не имеет фиксированных точек при четных $r>2$. Соответствующие $g_{1}, g_{2}$ инвариантные заряды, стартуя с различных начальных значений, пересекают область устойчивости $(5)$ действия $S_{\mathrm{e}}$ приблизительно по одной траектории. Уход траектории за границу области устойчивости обычно интерпретируется как указание на существование фазового перехода первого рода.

Известно, что существуют модели, в которых учет старших порядков теории возмущений существенно влияет на анализ устойчивости фиксированных точек. В качестве примеров можно привести так называемую С-модель критической динамики [5], киральные трехмерные модели и разнообразные спиновые системы с анизотропией [6]-[11]. Поэтому естественным представляется вопрос, не возникнет ли аналогичная ситуация в рассматриваемом случае.

В разделе 2 представлены результаты вычислений РГ-функций в трехпетлевом приближении. Мы решили не заниматься поиском и анализом фиксированных точек уравнения РГ в данном приближении, ограничившись анализом поведения фазовых траекторий. Как указано в работе [1], не только положение фиксированных точек и соответствующие критические индексы, но и сами уравнения на инвариантные заряды можно построить в форме стандартного є-разложения, т. е. с малым параметром - величиной отклонения размерности пространства от логарифмической размерности теории $(\epsilon=4-D)$.

Для анализа фазового портрета этих уравнений в физических размерностях пространства $(\epsilon=1, \epsilon=2)$ на предмет выхода за границу (5) области устойчивости $S_{\mathrm{e}}$ необходимо проводить их пересуммирование, например, по Борелю. Такое пересуммирование требует знания асимптотики высоких порядков (АВП) $\epsilon$-разложения. АВП найдена методами инстантонного анализа [12] в разделе 3 . В рассматриваемой модели обнаружено существование нескольких инстантонов различной матричной структуры. Их структура оказывается существенной для борелевского пересуммирования при различных соотношениях зарядов $g_{1}$ и $g_{2}$. 
В разделе 4 проведено пересуммирование и решение уравнений РГ на инвариантные заряды. Численный анализ этих уравнений, проведенный в разделе 5, показывает, что характер поведения инвариантных зарядов принципиально не меняется по сравнению с результатами однопетлевых вычислений как до границы устойчивости, так и в достаточно широкой области за пределами устойчивости системы. Тем самым установлено, что фазовый переход в сверхпроводящее состояние в фермионных системах, в которых носителям заряда соответствует $r \geqslant 4$, скорее всего, является переходом первого рода и имеет место при более высоких температурах, чем это предсказывается традиционной теорией [2].

\section{2. РГ-АНАЛИЗ}

Сформулируем основные правила диаграммной техники для теории с действием (4). Отличие от скалярной модели имеется лишь в симметрийных коэффициентах, петлевые интегралы здесь те же, что и в обычной скалярной теории $\phi^{4}$, их значения приведены, например, в [5]. Затравочный пропагатор для модели в представлении Фурье

$$
\begin{aligned}
\Pi_{i_{1} i_{2}}^{j_{1} j_{2}} & =\frac{W_{i_{1} i_{2}}^{j_{1} j_{2}}}{\mathbf{k}^{2}+\tau} \\
W_{i_{1} i_{2}}^{j_{1} j_{2}} & =\frac{1}{2}\left(\delta_{i_{1} j_{1}} \delta_{i_{2} j_{2}}-\delta_{i_{1} j_{2}} \delta_{i_{2} j_{1}}\right),
\end{aligned}
$$

где $\delta_{i k}-$ символ Кронекера. Тензор $W_{i_{1} i_{2}}^{j_{1} j_{2}}$ антисимметричен по перестановкам $i_{1} \leftrightarrow$ $i_{2}$ и $j_{1} \leftrightarrow j_{2}$ и симметричен относительно перестановок $\left(i_{1}, i_{2}\right) \leftrightarrow\left(j_{1}, j_{2}\right)$. Графические изображения тензорных структур вершин и пропагатора представлены на рис. 1 (антисимметризация вершинных множителей подразумевается).
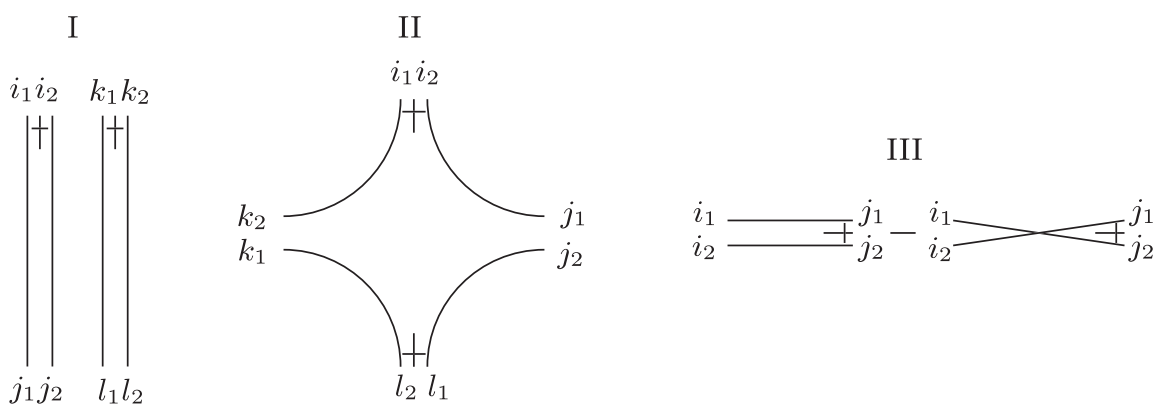

Рис. 1. Тензорные структуры вершин и пропагатора. Вершина I соответствует $g_{1}$, вершина II - $g_{2}$, вершина III - пропагатору.

В работе используется размерная регуляризация в пространстве $4-\epsilon$, а в качестве схемы ренормировок - MS-схема, в которой константы ренормировки имеют вид

$$
Z=1+\frac{Z^{1}}{\epsilon}+O\left(\frac{1}{\epsilon^{2}}\right)
$$


где $Z^{1}$ обозначает вычет в простом полюсе по $\epsilon$ соответствующей константы ренормировки, а РГ-функции в MS-схеме имеют вид

$$
\beta_{j}=-g_{j}\left(\epsilon+\gamma_{g_{j}}\right), \quad \gamma_{g_{j}}=-g_{k} \partial_{k} Z_{g_{j}}^{1}
$$

Трехпетлевой расчет бета-функций был выполнен на основе известных значений расходимостей графов [5]. Для вычисления тензорных сверток мы использовали программу FORM [13]. В результате получено

$$
\begin{aligned}
& \beta_{1}=-\epsilon g_{1}+\frac{1}{4}\left(r^{2}-r+8\right) g_{1}^{2}+(r-1) g_{1} g_{2}+\frac{3}{4} g_{2}^{2}- \\
& -\frac{9}{48}\left(3 r^{2}-3 r+14\right) g_{1}^{3}-\frac{11}{4}(r-1) g_{1}^{2} g_{2}-\frac{1}{32}\left(5 r^{2}-15 r+92\right) g_{1} g_{2}^{2}- \\
& -\frac{3}{8}(r-2) g_{2}^{3}+\frac{1}{512}\left[33 r^{4}-66 r^{3}+(955+480 \zeta(3)) r^{2}-(480 \zeta(3)+922) r+\right. \\
& +2960+2112 \zeta(3)] g_{1}^{4}+\frac{1}{128}\left[79 r^{3}-158 r^{2}+(1397+768 \zeta(3)) r-1318-\right. \\
& -768 \zeta(3) r] g_{1}^{3} g_{2}+\frac{1}{1024}\left[3 r^{4}-12 r^{3}+(576 \zeta(3)+3355) r^{2}-\right. \\
& -(1728 \zeta(3)+7568) r+9216 \zeta(3)+14788] g_{1}^{2} g_{2}^{2}+\frac{1}{512}\left[60 r^{3}-321 r^{2}+\right. \\
& +(2943+1152 \zeta(3)) r-2304 \zeta(3)-4092] g_{1} g_{2}^{3}+\frac{1}{1024}\left[(96 \zeta(3)+193) r^{2}-\right. \\
& -(576 \zeta(3)+891) r+1536 \zeta(3)+1860] g_{2}^{4}, \\
& \beta_{2}=-\epsilon g_{2}+\frac{1}{4}(2 r-5) g_{2}^{2}+3 g_{1} g_{2}- \\
& -\frac{3}{32}\left(r^{2}-7 r+20\right) g_{2}^{3}-\frac{1}{4}(11 r-20) g_{2}^{2} g_{1}-\frac{1}{16}\left(5 r^{2}-5 r+82\right) g_{1}^{2} g_{2}+ \\
& +\frac{1}{1024}\left[26 r^{3}-(383+96 \zeta(3)) r^{2}+(2459+1152 \zeta(3)) r-4060-2688 \zeta(3)\right] g_{2}^{4}+ \\
& +\frac{1}{128}\left[(96 \zeta(3)+182) r^{2}-(963+576 \zeta(3)) r+1536 \zeta(3)+1937\right] g_{2}^{3} g_{1}+ \\
& +\frac{1}{512}\left[-70 r^{3}+11 r^{2}+(6423+4608 \zeta(3)) r-8064 \zeta(3)-10366\right] g_{1}^{2} g_{2}^{2}+ \\
& +\frac{1}{256}\left[-13 r^{4}+26 r^{3}+(192 \zeta(3)+355) r^{2}-(368+192 \zeta(3)) r+\right. \\
& +3284+2688 \zeta(3)] g_{1}^{3} g_{2},
\end{aligned}
$$

где сделана замена $g_{i} \rightarrow g_{i} / 16 \pi^{2}$. Результаты вычислений были проверены по совпадению с известными результатами вычисления [5] $\beta$-функций $O(r)$-симметричной $\phi^{4}$-модели при $r=2,3$.

Поскольку в однопетлевом приближении не удалось ни обнаружить ИК-устойчивых фиксированных точек, ни привести аналитического решения для инвариантных 
зарядов при $r>2$ [1], мы в дальнейшем используем численные решения полученной системы уравнений на инвариантные заряды.

Сделаем растяжение динамической переменной $\xi=\ln \left(\tau / M^{2}\right)$ и зарядов: $g_{i} \rightarrow \epsilon g_{i}$, $\xi \rightarrow \xi / \epsilon(M-$ ренормировочная масса, ренормированные заряды связаны с "затравочными" соотношениями $g_{i}^{0}=M^{\epsilon} g_{i} Z_{g i}$ ). После этого уравнения на инвариантные заряды можно записать в виде разложения по $\epsilon$ :

$$
\begin{gathered}
\partial_{\xi} \bar{g}_{i}=-\bar{g}_{i}+\sum_{N=0}^{K} \epsilon^{N} \beta_{i}^{(N)}\left(\bar{g}_{1}, \bar{g}_{2}\right), \quad i=1,2, \\
\left.\bar{g}_{i}\right|_{\xi=0}=g_{i} .
\end{gathered}
$$

Явные выражения для $\beta_{i}^{(N)}\left(g_{1}, g_{2}\right)$ определены соответствующими порядками разложений (7), (8). В рассматриваемом случае трехпетлевого приближения $K=2$. Результаты однопетлевого счета соответствуют рассмотренным в работе [1] вкладам $\sim \epsilon^{0}$.

Для исследования уравнений типа (9) при физических значениях $\epsilon=1$ и $\epsilon=2$, т. е. с большим параметром разложения, воспользуемся пересуммированием имеющихся членов уравнений. Такое пересуммирование реализуется с помощью преобразования Бореля, которое требует знания АВП разложения (9), т. е. информацию о поведении $\beta_{i}^{(N)}\left(g_{1}, g_{2}\right)$ при $N \rightarrow \infty$.

\section{3. АСИМПТОТИЧЕСКИЙ АНАЛИЗ}

Нас интересует АВП разложений в модели (4) по паре зарядов $g_{1}$ и $g_{2}$. Эта задача не является новой, решение похожей проблемы можно найти в работе [14], где исследовались АВП динамических моделей, в частности, с несколькими константами взаимодействия. Проблема упрощается тем, что реально нас интересует не АВП поведения по каждому заряду в отдельности, а поведение разложения по числу петель, в котором заряды можно считать пропорциональными.

Сделав переобозначение $g_{i} \rightarrow z g_{i}$ в действии (4), будем исследовать разложение по степеням $z$. По окончании вычислений АВП положим $z=\epsilon$ и учтем, что $\beta_{i}^{(N)}\left(g_{1}, g_{2}\right)$ следует поделить на $\epsilon$ для построения $\epsilon$-разложений в уравнениях (9).

В настоящем разделе используется подход, предложенный в работе [15], который специально разработан для исследования АВП констант ренормировки в MS-схеме. Рассматриваются частично-ренормированные функции Грина, в которых осуществлены вычитания на расходимости всех подграфов вплоть до порядка $N-1$. Сами ультрафиолетовые расходимости в функциях Грина в MS-схеме имеют вид полюсов по $\epsilon$.

Нахождение АВП коэффициентов разложения $2 k$-хвостых ренормированных функций Грина в ряд по переменной z основывается на интегральной формуле Коши [12]

$$
G_{2 k}^{(N)}\left(x_{1}, \ldots, x_{2 k}\right)=\frac{(-1)^{N}}{2 \pi i} \oint_{\gamma} \frac{d z G_{2 k}\left(z, x_{1}, \ldots, x_{2 k}\right)}{(-z)^{N+1}}
$$


с последующим применением метода перевала. Здесь $\gamma$ - замкнутый контур в комплексной плоскости вокруг нуля и

$$
\begin{gathered}
G_{2 k}\left(z, x_{1}, \ldots, x_{2 k}\right)=W^{-1} \int \mathcal{D} \chi \mathcal{D} \chi^{+} \chi\left(x_{1}\right) \chi^{+}\left(x_{2}\right) \ldots \chi\left(x_{2 k-1}\right) \chi^{+}\left(x_{2 k}\right) e^{-S_{\mathrm{R}}}, \\
W=\int \mathcal{D} \chi \mathcal{D} \chi^{+} e^{-S_{\mathrm{R}}}
\end{gathered}
$$

$S_{\mathrm{R}}$ - ренормированное действие (4). АВП, как обычно [12], будем искать при $\tau=0$ и $D=4$. После подстановки (11) в (10) и растяжения переменных $g_{i} \rightarrow g_{i} / N M^{\epsilon}$, $\chi \rightarrow \sqrt{N} \chi, \chi^{+} \rightarrow \sqrt{N} \chi^{+}$вариационные уравнения для функционала $J=S_{\mathrm{R}}+\ln (-z)$ принимают вид

$$
\begin{gathered}
-\Delta \chi+\frac{z g_{1}}{2} \chi \operatorname{Tr} \chi \chi^{+}+\frac{z g_{2}}{2} \chi \chi^{+} \chi=0 \\
-\Delta \chi^{+}+\frac{z g_{1}}{2} \chi^{+} \operatorname{Tr} \chi \chi^{+}+\frac{z g_{2}}{2} \chi^{+} \chi \chi^{+}=0 \\
\int d \mathbf{x}\left\{\frac{z g_{1}}{4}\left(\operatorname{Tr} \chi \chi^{+}\right)^{2}+\frac{z g_{2}}{4} \operatorname{Tr} \chi \chi^{+} \chi \chi^{+}\right\}=-1
\end{gathered}
$$

Учет контрчленов $Z_{i}-1$ в вариационных уравнениях приводит лишь к поправкам порядка $1 / N$ к решениям системы (12) (см. [12], [15]). Первые два уравнения различаются эрмитовым сопряжением, поэтому достаточно рассматривать лишь одно из них. Кроме того, без ограничения общности можно считать, что антисимметричные поля $\chi$ и $\chi^{+}$приведены к пфаффовой форме, т. е. имеют вид блочно-диагональных матриц с $p=r / 2$ блоками

$$
\chi=\left(\begin{array}{cccc}
s_{1} \sigma & 0 & \ldots & 0 \\
0 & s_{2} \sigma & \ddots & \vdots \\
\vdots & \ddots & \ddots & 0 \\
0 & \ldots & 0 & s_{p} \sigma
\end{array}\right), \quad \chi^{+}=\left(\begin{array}{cccc}
-s_{1}^{*} \sigma & 0 & \ldots & 0 \\
0 & -s_{2}^{*} \sigma & \ddots & \vdots \\
\vdots & \ddots & \ddots & 0 \\
0 & \ldots & 0 & -s_{p}^{*} \sigma
\end{array}\right)
$$

где

$$
\sigma=\left(\begin{array}{cc}
0 & -1 \\
1 & 0
\end{array}\right)
$$

Также предполагается, что функции $s_{j}(\mathbf{x})$, где $j=1, \ldots, p$, принимают значения в комплексной плоскости, т. е. $s_{j}: \mathbb{R}^{D} \rightarrow \mathbb{C}$. Дифференциальные уравнения (12) сводятся к системе дифференциальных уравнений на функции $s_{j}(\mathbf{x})$ :

$$
-\Delta s_{i}(\mathbf{x})+z g_{1} \sum_{k=1}^{p}\left|s_{k}(\mathbf{x})\right|^{2} s_{i}(\mathbf{x})+\frac{z g_{2}}{2}\left|s_{i}(\mathbf{x})\right|^{2} s_{i}(\mathbf{x})=0
$$


Для построения решений этой системы при $D=4$ выберем известный анзац

$$
s_{i}(\mathbf{x})=\frac{\alpha_{i} y^{-1}}{\left|\mathbf{x}-\mathbf{x}_{0}\right|^{2}+y^{2}}, \quad \alpha_{i} \in \mathbb{C},
$$

пропорциональный решению аналогичных уравнений для скалярной теории $\phi^{4}[12]$. Произвольные параметры $y, \mathbf{x}_{0}$ - радиус и центр инстантона соответственно - отражают дилатационную и трансляционную инвариантность теории. При вычислениях эта инвариантность, как и в работе [12], учитывается с помощью трюка Фаддеева-Попова, который фиксирует произвол для каждого инстантона $\left(\mathbf{x}_{0}=0\right.$, $y=1)$ и вводит интегрирования с корректной мерой по $\mathbf{x}_{0}, y$. Подстановка формулы (16) в уравнения (15) приводит к системе алгебраических уравнений на константы $\alpha_{i}$ :

$$
8 \alpha_{i}+z g_{1} \sum_{k=1}^{p}\left|\alpha_{k}\right|^{2} \alpha_{i}+\frac{z g_{2}}{2}\left|\alpha_{i}\right|^{2} \alpha_{i}=0
$$

Видно, что нетривиальными решениями являются инстантоны, у которых может быть $m=0, \ldots, p-1$ нулевых блоков с $\left|\alpha_{i}\right|=0$ и $n=p, \ldots, 1$ ненулевых блоков с $\left|\alpha_{i}\right|^{2}=-16 /\left(2 n z g_{1}+z g_{2}\right)$, причем $n+m=p$. Фаза чисел $\alpha_{i}$ не фиксируется и является произвольным параметром наряду с $\mathbf{x}_{0}$ и $y$. Кроме произвольных параметров $y, \mathbf{x}_{0}$ и $n$ фазовых параметров существует инвариантность относительно унитарных преобразований $U(r)$, с помощью которых матрицы были приведены к пфаффовой форме. Тем самым общее число нулевых мод определяется выражением $r^{2}-2 r+n+5$, что влияет на показатель степени $N$ в исследуемой АВП. Для установления точки стационарности $z_{\text {st }}$ воспользуемся интегральным условием (12), откуда получаем

$$
z_{\mathrm{st}}(n)=-\frac{4 n}{3} \frac{1}{2 n g_{1}+g_{2}} .
$$

Определение АВП функций Грина по известным инстантону (16) и стационарному значению параметра разложения (18) является стандартной процедурой [12], [15]. Как и в работе [15], по рассчитанной методом инстантонного анализа АВП вычета в простом полюсе $G_{4}^{1(N)} / \epsilon$ была определена АВП разложений $\beta$-функций рассматриваемой теории

$$
\beta_{i}^{(N)}\left(g_{1}, g_{2}\right)=\text { const }_{i} \cdot N ! N^{b_{n}}(-a)^{N}\left(1+O\left(N^{-1}\right)\right),
$$

где const ${ }_{i}$ - некоторые числовые константы, $b_{n}=\left(r^{2}-2 r+n+11\right) / 2$ и $a=\max _{n}|a(n)|$, $a(n)=-1 / z_{\mathrm{st}}(n)$. Из равенства $(18)$ видно, что $a(n)$ меняются вместе с $g_{i}$, поэтому главный вклад в асимптотику (19) дает наибольшее по модулю значение $a(n)$, остальные вносят экспоненциально малый вклад и должны быть отброшены. Результат (19) показывает, что ряд в правой части (9) является расходящимся в классическом смысле, поэтому при учете многопетлевых вкладов необходимо использовать методы пересуммирования. Вследствие факториального роста коэффициентов наиболее эффективным является метод Бореля или его обобщение - метод Бореля-Леруа. 


\section{4. ПЕРЕСУММИРОВАНИЕ}

Общая идеология пересуммирования по Борелю-Леруа [16], [17] заключается в следующем: пусть некая величина, в нашем случае $\beta(z)_{i}$, представима степенным рядом по $z$ с коэффициентами, имеющими при $N \rightarrow \infty$ вид (19),

$$
\beta(z)_{i}=\sum_{N \geqslant 0} z^{N} \beta_{i}^{(N)},
$$

тогда ей сопоставляется функция $B(z)$, определяемая следующим равенством:

$$
B(z)_{i}=\sum_{N \geqslant 0} B_{i}^{(N)} z^{N}, \quad B_{i}^{(N)}=\frac{\beta_{i}^{(N)}}{\Gamma\left(N+b_{0}+1\right)},
$$

где $\Gamma(N)$ - гамма-функция Эйлера, $b_{0}$ - произвольный параметр. Обратное преобразование, позволяющее восстановить функцию $\beta(z)_{i}$ по ее образу $B(z)$, задано формулой

$$
\beta(z)_{i}=\int_{0}^{\infty} d t e^{-t} t^{b_{0}} B(z t)_{i} .
$$

Таким образом, знание асимптотики (19) вместе с некоторыми предположениями об аналитических свойствах функции $B(z)$ позволяет пересуммировать ряд (20). Нетрудно заметить, что при $N \rightarrow \infty$ коэффициенты $B^{(N)} \sim(-a)^{N} N^{b_{n}-b_{0}}$. Это означает, что такой ряд сходится в круге, радиус которого определяется величиной $a$, следовательно, функция $B(z)$ является аналитической в этой области.

Известны разные схемы преобразования Бореля-Леруа, использующие различные параметры в качестве подгоночных [16], [17]. Мы будем использовать так называемую схему конформного преобразования Бореля-Леруа, как наиболее точно контролируемую с точки зрения знания АВП. Она содержит минимум свободных параметров и приводит к наиболее точным результатам (см., например, [18]). Особенность нашей задачи заключается в том, что положение полюсов борелевского образа исследуемых $\beta$-функций зависит от соотношения зарядов $g_{1}$ и $g_{2}$, т. е. от конкретной точки в плоскости $\left(g_{1}, g_{2}\right)$, в которой рассматривается система.

Рассмотрим случай $r=4$, что физически соответствует, например, фермионам со спином $3 / 2$. Существуют два типа инстантонов: с одним ненулевым блоком $a(1)=$ $(3 / 4)\left(2 g_{1}+g_{2}\right)$ и с двумя ненулевыми блоками $a(2)=(3 / 8)\left(4 g_{1}+g_{2}\right)$. С эволюцией инвариантных зарядов согласно уравнениям (9) параметры $a(1), a(2)$ меняются не только по абсолютной величине, но и по знаку. Им соответствуют разные области в плоскости $\left(g_{1}, g_{2}\right)$, с различными свойствами сходимости ряда для $B(z)$.

Область I: если инвариантные заряды удовлетворяют условию $8 \bar{g}_{1}+3 \bar{g}_{2}>0$, то $|a(1)|>|a(2)|$. Ближайшая к нулю сингулярность располагается в точке $z=-R_{1}=$ $-1 / a(1)$.

Область II: если инвариантные заряды удовлетворяют условию $8 \bar{g}_{1}+3 \bar{g}_{2} \leqslant 0$, то $|a(2)|>|a(1)|$. В этом случае ближайшая сингулярность располагается уже на положительной полуоси в точке $z=R_{2}=1 /|a(2)|$. 
Таким образом, вся плоскость $\left(\bar{g}_{1}, \bar{g}_{2}\right)$ разделена прямой $8 \bar{g}_{1}+3 \bar{g}_{2}=0$. Выше этой границы аналитические свойства борелевского образа определяются наиболее вырожденным инстантоном, т. е. инстантоном с одним ненулевым блоком, а ниже границы - невырожденным инстантоном.

Пусть инвариантные заряды находятся в области I. Именно здесь находятся физические стартовые значения инвариантных зарядов, т. е. ренормированные заряды модели (4). При этом контур интегрирования в (22) пересекает область аналитичности функции $B(z)$ в точке $R_{1}$. Проблема аналитического продолжения во внешность круга сходимости решается методом конформного отображения [16], [17].

Обычно конформное отображение комплексной плоскости выбирают в виде [16]

$$
u(z)=\frac{\sqrt{1+a z}-1}{\sqrt{1+a z}+1} \quad \Leftrightarrow \quad z(u)=\frac{4 u}{a(u-1)^{2}} .
$$

Равенство $u(z)=O(z)$ при $z \rightarrow 0$ позволяет разложить ряд (21) по переменной $u$ :

$$
\begin{gathered}
B(z)_{i}=\sum_{N \geqslant 0} B_{i}^{(N)} z^{N}=\sum_{N \geqslant 0} U_{i}^{(N)} u^{N}, \\
U_{i}^{(0)}=B_{i}^{(0)}, \quad U_{i}^{(N)}=\sum_{m=1}^{N} B_{i}^{(m)}\left(\frac{4}{a}\right)^{m} C_{N+m-1}^{N-m}, \quad N \geqslant 1,
\end{gathered}
$$

тогда конформное преобразование Бореля приобретает вид

$$
\beta(z)_{g_{i}}=\sum_{N \geqslant 0} U_{i}^{(N)} \int_{0}^{\infty} d t t^{b_{0}} e^{-t} u(z t)^{N} .
$$

Произвольный параметр пересуммирования $b_{0}$ из соображения ускорения сходимости рядов принято фиксировать условием $b_{0}=b_{n}+3 / 2$ [16], [17].

\section{5. ЧИСЛЕННЫЙ АНАЛИЗ}

Пользуясь полученной асимптотической формулой (19) для коэффициентов $\epsilon$-разложения и выражением (25), можно окончательно написать пересуммированные РГ-уравнения на инвариантные заряды:

$$
\begin{gathered}
\partial_{\xi} \bar{g}_{i}=-\bar{g}_{i}+\sum_{N=0}^{K} U_{i}^{(N)} \int_{0}^{\infty} d t t^{b_{0}} e^{-t} u(\epsilon t)^{N}, \\
\left.\bar{g}_{i}\right|_{\xi=0}=g_{i} .
\end{gathered}
$$

Отметим, что в рассматриваемом случае $U_{i}^{(N)}$ и $u(t)$ являются функциями $\bar{g}_{i}$.

Численное решение системы (26) осуществлялось следующим образом. Выбрав начальную точку в плоскости зарядов $\left(g_{1}, g_{2}\right)$, определяем соответствующий радиус сходимости или значение $a$ и производим в соответствии с изложенным в разделе 4 пересуммирование правых частей уравнений (26). Затем, пользуясь конечностно-разностным методом, находим новые значения инвариантных зарядов, для которых повторяем процедуру пересуммирования. И так далее. 


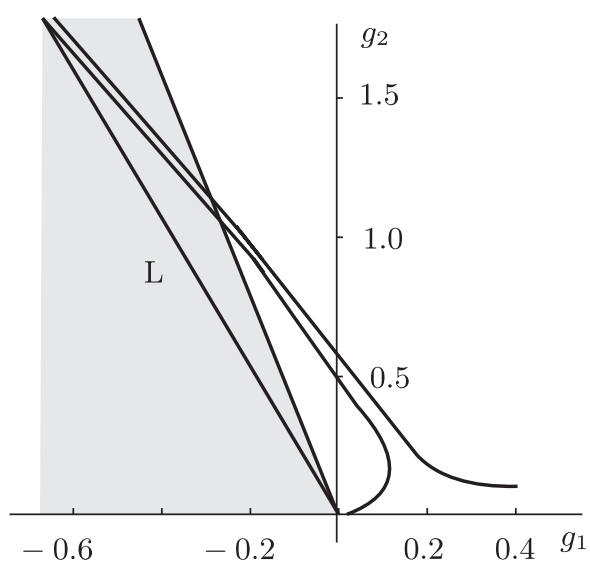

a

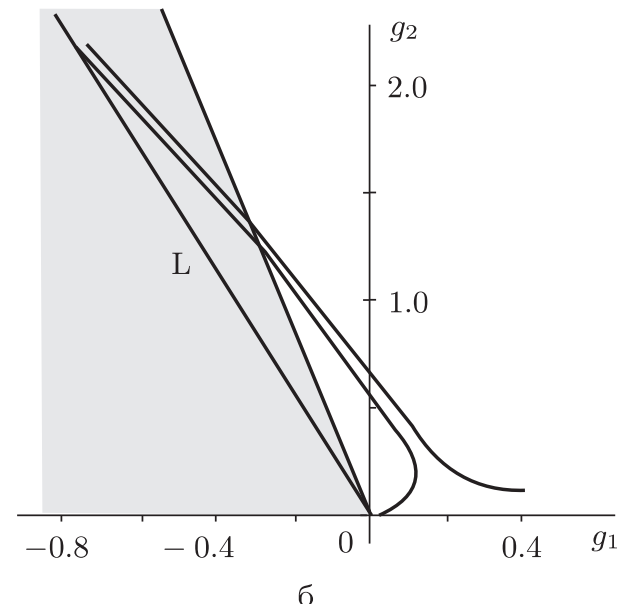

6

Рис. 2. Траектории инвариантных зарядов с различными начальными условиями при $D=3$ (а) и $D=2$ (б). Линии $L$ соответствуют $8 \bar{g}_{1}+3 \bar{g}_{2}=0$. Заштрихованная часть соответствует области, в которой устойчивость действия нарушается.

В качестве примера исследовались решения системы (26) при $r=4$. На рис. 2 приведены две траектории, соответствующие различным начальным данным при $D=3$ (рис. $2 \mathrm{a}$ ) и при $D=2$ (рис. 2б). Область положительной определенности действия (4) не закрашена. При некотором значении $\xi_{0}$ траектории выходят из этой области практически по одной линии. Предъявленное на рисунках поведение слабо зависит от рассматриваемой размерности пространства. Численный анализ уравнений (26) при различных параметрах $r, \epsilon$ позволяет заключить, что и в трехпетлевом приближении в области (5) нами не обнаружено ИК-устойчивых фиксированных точек уравнения РГ. Кроме того, на уровне численного исследования видно, что решение задачи Коши (26) является устойчивым: небольшое варьирование начальных данных не приводит к существенному изменению поведения траекторий, поэтому здесь можно говорить о некой универсальности, несмотря на отсутствие в системе фиксированных точек. Полученные решения будут справедливы, пока верно представление (25), т. е. во всей области I. Далее, когда заряд попадает в область II, сингулярность $B(z)$ переходит на положительную полуось, т. е. на контур интегрирования. Это уже не позволяет использовать конформное преобразование Бореля в форме (25).

Сравнение результатов однопетлевого (1), двухпетлевого (2) и трехпетлевого (3) расчетов приведено на рис. За для $D=3$ и рис. $3 б$ для $D=2$. Из представленных графиков видно, что хотя трехпетлевые вычисления и не обеспечивают достаточно большую точность, особенно при $\epsilon=2$, тем не менее характер поведения траекторий с пересечением границы устойчивости системы неуклонно сохраняется.

Ситуация принципиально не меняется при $r>4$. Например, при $r=6$ в теории появляются уже три типа инстантонов. В этом случае плоскость $\left(g_{1}, g_{2}\right)$ будет делиться на большее число областей, чем при $r=4$. Отметим лишь, что до границы 


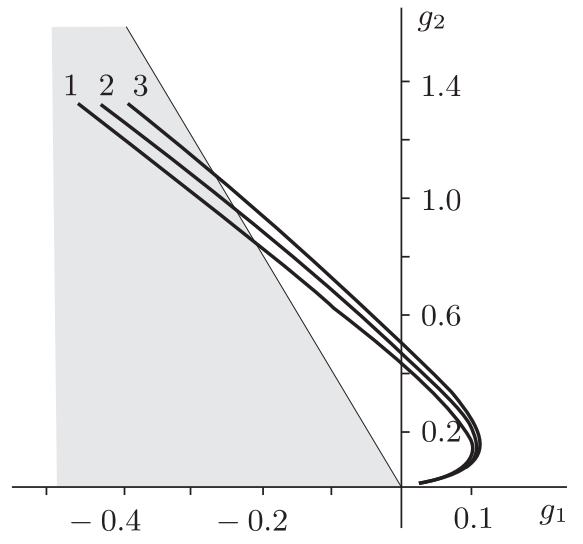

a

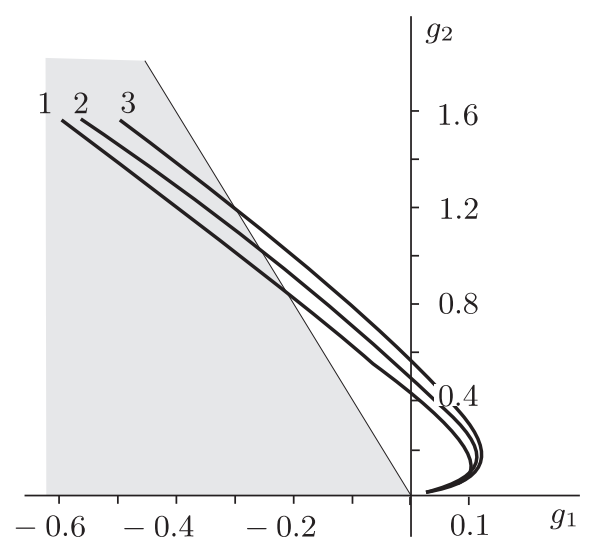

б

Рис. 3. Траектории инвариантных зарядов с различным числом учтенных петель диаграмм при $D=3$ (а) и $D=2$ (б).

устойчивости и в некоторой ее окрестности при любых $r$ главный вклад в асимптотику вносит наиболее вырожденный инстантон с $n=1$. Можно полагать, что если фазовый переход первого типа действительно имеет место, то он происходит вблизи границы устойчивости. По этой причине пересуммирование достаточно проводить лишь с $a=a(1)$.

\section{6. ЗАКЛЮЧЕНИЕ}

В работе проведено исследование АВП квантово-полевых разложений теории с взаимодействием типа $\phi^{4}$ с матричным антисимметричным полем. Найдены инстантоны. Обнаружена необычная ситуация, когда АВП меняется в зависимости от соотношения зарядов.

Анализ рассматриваемой модели нерелятивистских ферми-частиц с взаимодействием типа "плотность-плотность" и дополнительными индексами полей носителей зарядов (или связанных со старшими спинами, или обусловленных существованием различных подрешеток, или возникающих из-за вырождения зонной структуры) показывает, что приведенные в работе [1] результаты однопетлевого счета качественно не меняются при учете еще двух порядков теории возмущений. Инвариантные заряды по-прежнему выходят за границу области устойчивости действия, что, как и в [6]-[11], следует считать указанием на то, что в системе имеет место фазовый переход первого рода, причем при более высокой температуре, чем это предсказано общеизвестной теорией [2] для фазовых переходов второго рода.

Более того, результаты численного счета, как и в однопетлевом приближении [1], указывают на квазиуниверсальность (в смысле слабой зависимости от начальных данных) поведения фазовых траекторий и, следовательно, температуры фазового перехода. Это происходит несмотря на отсутствие ИК-устойчивых фиксированных точек уравнения РГ, влияющих на поведение в окрестности точки фазового перехода. Отметим, что упомянутые выше результаты получены для реальных размерностей пространства $D=3, D=2$. 
Мы надеемся более детально исследовать фазовый переход в рассматриваемой модели в последующих работах.

Благодарности. Работа выполнена при финансовой поддержке РФФИ (грант № 1.2-02-00874-а) и СПбГУ (грант № 11.38.636.2013).

\section{Список литературы}

[1] М. В. Комарова, М. Ю. Налимов, Ю. Хонконен, ТМФ, 176:1 (2013), 89-97.

[2] А. А. Абрикосов, Л. П. Горьков, И. Е. Дзялошинский, Методы квантовой теории поля в статистической физике, Добросвет, М., 2006.

[3] А.Н. Васильев, Функииональные методы в квантовой теории поля и статистике, Изд-во ЛГУ, Л., 1976.

[4] M. I. Katsnelson, Graphene: Carbon in Two Dimensions, Cambridge Univ. Press, Cambridge, 2012.

[5] А.Н. Васильев, Квантовополевая ренормгруппа в теории критического поведения и стохастической динамике, Изд-во ПИЯФ, СПб., 1998.

[6] P. Calabrese, P. Parruccini, A. I. Sokolov, Phys. Rev. B, 68:9 (2003), 094415, 8 pp.

[7] P. Calabrese, E. V. Orlov, P. Parruccini, A. I. Sokolov, Phys. Rev. B, 67:2 (2003), 024413, 9 pp., arXiv: cond-mat/0207187.

[8] P. Calabrese, P. Parruccini, A. I. Sokolov, Phys. Rev. B, 66:18 (2002), 180403, 4 pp., arXiv: cond-mat/0205046.

[9] P. Calabrese, P. Parruccini, A. I. Sokolov, Phys. Rev. B, 68:9 (2003), 094415, 8 pp., arXiv: cond-mat/0304154.

[10] S. A. Antonenko, A. I. Sokolov, K. B. Varnashev, Phys. Lett. A, 208:1-2 (1995), 161-164, arXiv: cond-mat/9803377.

[11] S. A. Antonenko, A.I. Sokolov, Phys. Rev. B, 49:22 (1994), 15901-15912, arXiv: cond-mat/9809306.

[12] Л. Н. Липатов, ЖЭТФ, 72:2 (1977), 411-427.

[13] J. A. M. Vermaseren, New features of FORM, arXiv: math-ph/0010025.

[14] J. Honkonen, M. V. Komarova, M. Yu. Nalimov, Nucl. Phys. B, 714:3 (2005), 292-306, arXiv: hep-th/0412256.

[15] М. В. Комарова, М. Ю. Налимов, ТМФ, 126:3 (2001), 409-426.

[16] J. Zinn-Justin, Quantum Field Theory and Critical Phenomena, Clarendon Press, Oxford, 1996.

[17] H. Kleinert, V. Schulte-Frohlinde, Critical Properties of $\phi^{4}$-Theories, World Sci., Singapore, 2001.

[18] М. Ю. Налимов, В. А. Сергеев, Л. Сладкофф, ТМФ, 159:1 (2009), 96-108.

Поступила в редакцию 17.05.2014, после доработки 29.05.2014 